\title{
Insights into the Structure-Property Relationship of Pharmaceutical Co-Crystals: Charge Density and Quantum Chemical Approaches
}

Lucy K. Mapp, ${ }^{\mathrm{a}, \mathrm{b}}$ Joseph Cadden, ${ }^{\mathrm{a}, \mathrm{b}}$ Wim T. Klooster, ${ }^{a}$ Srinivasulu Aitipamula ${ }^{\mathrm{b}}$ and Simon J. Coles ${ }^{\mathrm{a} *}$ ${ }^{a}$ Chemistry, Faculty of Engineering and Physical Sciences, University of Southampton, University Road, Southampton SO17 1BJ, U.K.

${ }^{\mathrm{b}}$ Formulated Products, Institute of Chemical and Engineering Sciences, A*STAR (Agency for Science, Technology and Research), 1 Pesek Road, Jurong Island, 627833, Singapore.

\section{Abstract}

A subset of co-crystal systems of the antipyretic and analgesic drug, propyphenazone, are used to probe the nature of the drug...co-former interactions. The experimental electron density distribution, based on very high-resolution single crystal diffraction, has been modelled and an analysis undertaken using Bader's Atoms in Molecules approach. Atomic charges, intermolecular interactions and their energies have been subsequently derived and compared between systems. Complementary theoretical calculations are used to derive interaction energies for intermolecular interactions beyond atom $\cdots$ atom contacts. These permit the deconvolution of the intermolecular interactions into their constituent energy components for a comprehensive analysis. This approach provides an insight into the factors affecting the assembly of the solid state, with the case of pharmaceutical co-crystals being highlighted in this work. Furthermore, this approach enables analysis of the effect of the co-former on various influencing factors that determine the physicochemical properties of these multi-component systems.

\section{Introduction}

Tailoring of molecular and crystal structure in order to modify materials' properties is a fundamental goal of crystal engineering.[1] Co-crystals[2] achieve this aim by the introduction of an additional molecular entity into the crystal lattice, which has the effect of altering the molecular arrangement, 3D packing and intermolecular interactions. Consequently, a range of physicochemical properties will also be altered.[3,4] Such properties include solubility,[5,6] stability,[7-9] crystallinity, melting 
point,[10] dissolution rate,[11] and mechanical properties.[12] Accordingly, co-crystals are a current research interest in a variety of fields where control of the solid state is key, including pharmaceuticals,[13] energetic materials[14] and agrochemicals.[15] The commonly used Aufbau Synthon approach to crystal engineering uses well-understood strong intermolecular interaction patterns to construct a crystal structure.[16] However, Parsons et al. identified 'the hazards of oversimplifying intermolecular interactions on the basis of prominent atom-atom contacts' as part of an analysis of the high-pressure polymorph of glycine.[17] There are very few approaches that can be used to relate physicochemical properties to crystal structure and this is a key deficiency in the ability to design particular materials possessing specific properties. Both electron density studies i.e. highly accurate X-ray diffraction studies that reveal the electron distribution in a crystal[18], and computational approaches can probe physicochemical properties from an electronic level, each using different methods. An experimental or theoretical electron density distribution (EDD) can provide quantitative analysis of intermolecular interactions, the identification of individual hydrogen bonds and derivation of properties. Thus their nature and strength can be determined. Computational techniques can be employed in parallel and provide supplementary data and complementary analyses. The combination of the data obtained from both these methods allows for comparisons to be made, and the trends observed rationalised.

The pharmaceutical industry in particular has embraced the potential of co-crystals, where inclusion in drug discovery programs and screening processes is widespread. FDA guidance now classifies them alongside traditional solid forms and states that 'a regulatory classification of a co-crystal is similar to that of a polymorph of the active pharmaceutical ingredient (API)' and therefore co-crystal drug products are now beginning to come to market.[19,20] Recent examples of these include Lexapro, an escitalopram oxalate and oxalic acid co-crystal, approved in 2009 for the treatment of depression and anxiety disorders,[21,22] and Entresto, a 2015 FDA approved sacubitril-valsartan drug-drug co-crystal used to treat heart failure. $[23,24]$ The ignificance of co-crystals to industry has therefore shifted from one of academic curiosity to true application and therefore a full understanding of their behaviour is fundamentally necessary.

A selection of our own work in this area includes a comprehensive co-crystal design, screen, and structural and physicochemical property analysis on an API with antipyretic and analgesic effects propyphenazone (1,5-dimethyl-2-phenyl-4-propan-2-yl-pyrazol-3-one, PROPY).[25] An interaction analysis of similar structures in the Cambridge Structural Database (CSD)[26] led to a co-crystal screen involving 90 co-formers which generated 15 putative new co-crystals and 8 novel co-crystal single crystal structures. Analysis of API...co-former interactions in the atomic resolution crystal structures 
led to some correlations with trends in physical properties such as stability, solubility and dissolution rate. PROPY is notable in that it possesses little classical hydrogen-bonding capability and therefore typical synthon-based design strategies were not plausible. Hence, a comprehensive screening process was performed. It was a goal of this study to use this constraint (limited hydrogen bonding functionality) to better understand the solid-state criterion for forming co-crystals. However, This type of study, however, affords a level of understanding that is often limited; trial and error approaches to co-crystal screening are often commonplace. The work presented herein builds on the knowledgebased design approach, described in the aforementioned work on PROPY co-crystal design; we propose a more detailed analysis of a carefully selected subset of the single crystal structures of cocrystals of PROPY (Figure 1). Such a closely related set of structures allows a constrained analysis where particular factors can be compared and rationalised.

Quantum Crystallography is a concept that integrates quantum chemical methods with the fundamental, underlying principles of crystallography determined by the electron density and its distribution.[27] The advantages of this are twofold in that these studies provide quantitative analysis of the systems at the electronic level, and hence changes in molecular charge distribution between different crystals can be assessed. This also permits a deeper and extended investigation of the intermolecular interactions, the associated energies and components thereof. Co-crystals and their analysis provide an ideal vehicle to explore this methodology due to their reliance on multiple components being non-covalently bound together in the crystal lattice; the structural effect on various physicochemical properties can therefore be thoroughly investigated. 
<smiles>O=C(O)c1ccc(O)cc1</smiles><smiles>O=C(O)/C=C/C(=O)O</smiles>

\section{4-hydroxybenzoic acid (4HBA)}

fumaric acid (FA)<smiles>Oc1ccc(O)cc1</smiles>

hydroquinone $(\mathrm{HQ})$<smiles>Cc1c(C(C)C)c(=O)n(-c2ccccc2)n1C[R6](=O)O[Na]</smiles>

methylparaben (MPAR)

Figure 1. Molecular structure of PROPY and the four co-formers chosen for this study.

In our aforementioned PROPY study, the whole system is initially assessed to provide a holistic view of the structure and all the molecular pair interaction energies. This is in alignment with Desiraju and Nangia's view that a crystal structure must be assessed and visualised as a whole entity, rather than focussing on localised, specific interactions.[28]

Herein we combine both theoretical and experimental approaches to analyse individual interaction energies of a structurally-related sub-set of PROPY co-crystals. Interactions are hierarchically ranked based on the energies and components thereof, giving rise to three sets of interactions for each cocrystal system: strong hydrogen-bonds, moderate favourable interactions, and much weaker (and sometimes not all favourable) interactions. These are related to the assessed physicochemical properties of each system, in order to provide greater insight into the structure-property relationships. Whilst structurally similar, the chosen sub-set of structures display sufficient diversity for a selection of variables, in addition to the primary $\mathrm{O}-\mathrm{H} \cdots \mathrm{O}$ hydrogen bond, to be investigated. These include stoichiometry and aromaticicty as well as the nature of the primary hydrogen bond donor group functionality $\left(\mathrm{O}-\mathrm{H}_{\text {hydroxyl }} \cdots \mathrm{O}\right.$ vs. $\left.\mathrm{O}-\mathrm{H}_{\text {carboxylic acid }}\right)$. 


\section{Experimental Methods}

\subsection{High Resolution Single Crystal X-Ray Diffraction}

Data was collected for all four systems on the Rigaku FRE+ diffractometer equipped with VHF Varimax confocal mirrors, an AFC10 goniometer and an HG Saturn724+ detector using Mo-Ka radiation $(\lambda=$ 0.71075 Á). Crystal Clear V3.1[29] software was used for data collection and CrysAlisPro for data reduction and Gaussian absorption correction. All data sets were collected at $100 \mathrm{~K}$ and suitable crystals were selected and affixed to a MiTeGen MicroMesh support using two-component glue. This was cooled for 5-10 minutes before centering to ensure no crystal contraction or movement after centering occurred. For all systems an initial short screening data collection was completed using the attenuator for overload corrections. The experimental parameters were: exposure, 1 second; redundancy, $3-4$; resolution, $0.7 \AA$. Following this a full high-resolution data collection was calculated, with the same crystal and in the same orientation. The main experimental parameters for highresolution data collection were: exposure, 1 second (low angle) and 40-50 seconds (high angle); redundancy, 9-20; resolution, 0.41 Å. Full and comprehensive details of high-resolution data collection parameters can be found in the Supporting Information Section 1.1.1, Table S1. CCDC deposition numbers for the four co-crystals are: 1976434, 1976435, 1976453, 1976484.

\subsection{Experimental Electron Density Distributions}

\subsubsection{Atomic Interaction Energies}

The involvement of electrons in chemical bonds cause the atoms involved to become non-spherical, and a multipole model can be used to model this atomic non-sphericity.[30] In this approach (which is described thoroughly in this reference) the electron density is split into the spherical core electron density, the spherical valence electron density and the deformation valence density, the population, expansion and contraction of which can be subject to (multipole) refinement. EDDs obtained from a multipole model refinement of high resolution experimental diffraction data enable the analysis of specific atom $\cdots$ atom interactions. Quantitative information can be derived for all interactions, both intra- and inter-molecular and can be classified based on topological properties of the electron density at critical points. It has been shown that summing all experimental interaction energies between two entities, as used in the Espinosa-Molins-Lecomte (EML) scheme,[31] underestimates the total energy in comparison to that generated by the PIXEL method,[32] however this enables relative energies to be determined and a ranking of strength and importance to be ascertained. 
Full details of theoretical calculations and experimental data collection, multipole refinement and EDD analyses are provided as Supporting Information. Experimental parameters are detailed in Table S1 of the Supporting Information (see sections 1.1-1.3), while theoretical calculations are in Tables S12-13.

\subsection{Molecular Pair Interaction Energies}

The calculation of intermolecular interaction energies detailed by Spackman et al. and implemented in CrystalExplorer (CE) provides an overview of molecule $\cdots$ molecule interactions of a system.[33] Gaussian[34] B3LYP/6-31G(d,p) monomer wave functions for each molecule are determined within the software, from which the interaction energies are calculated.[35] Each interaction energy can be deconstructed into its four constituent terms $E_{\text {coul }}$ (coulombic), $E_{\text {pol }}$ (polarisation), $E_{\text {disp }}$ (dispersion) and $E_{\text {rep }}$ (repulsion). Hirshfeld surfaces[36-40] can be transformed into a 2D representation, a fingerprint plot, and numerical data extracted to compare the relative proportions of different contact types in each structure. Gavezzotti's PIXEL approach[41-44] is similar to that used by CE but separates interaction types into energies of $A \cdots A, A \cdots B, B \cdots A$ and $B \cdots B$ interactions (considering both donors and acceptors for a two-component system). These computational methods offer a quantitative assessment at the molecule $\cdots$ molecule level and can identify the strongest, and possibly most important, interactions in a system. By comparison and combination of these two approaches a comprehensive overview of the pairwise interactions can be derived.

A comparison of the energy components derived from both the PIXEL and CrystalExplorer methods shows them to be very similar (Tables S12 and S13). While both methods were employed throughout, the majority of theoretical data presented herein are from calculations using the PIXEL method as it readily specifies the molecules involved in an interaction making it easier to distinguish and group: PROPY...PROPY, PROPY...co-former or co-former...co-former interactions. The exception to this is when specific types of theoretical interaction energies are discussed, e.g. $\mathrm{C}-\mathrm{H}, \mathrm{O}-\mathrm{H}$, where CrystalExplorer energy components and fingerprint plots are used.

\section{Results}

Analysis of classical non-covalent interactions alone provides only a generalised and simplified approach to rationalising the nature of the solid state. Since the introduction of high resolution EDD analysis considerably more detail about these interactions can be derived, however this still does not represent the whole range of interactions between molecules in crystals. We present analysis that begins with EDD analysis of classical interactions, but moves on to consider the total interaction present between neighbouring whole molecules in the crystal. These molecule $\cdots$ molecule interactions 
can be broken down into the wider range of constituent components which can be related back to a variety of classical interactions, such as hydrogen bonds, $\pi-\pi$ and electrostatics. The interaction analysis results presented here form the basis of the rationalisation of co-crystal composition and physical properties from a hierarchical ranking of these interaction energies.

\subsection{Intermolecular Interactions From EDDs}

When the experimental EDD is considered, pairs of molecules can be identified due to being linked by a critical point. Figure 2 shows the total interaction energies (calculated by PIXEL) for molecule...molecule pairs formed between all components in the four systems studied. We now discuss the relative energy of molecular pairs in these scatter plots in the context of the classical interactions observed from the experimental EDD.

Each molecule-molecule pairing in all four co-crystals is dominated by a single, strong hydrogen bond (seen towards the bottom of each plot, $>40 \mathrm{kJmol}^{-1}$ ) arising from the interaction between PROPY and co-former via the $\mathrm{C}=\mathrm{O}$ functionality, The exception to this is PROPY-4HBA which contains a strong interaction between a co-former $\cdots$ co-former molecular pair in addition. This arises from the carboxylic acid dimer which is present. PROPY-HQ also displays strong co-former $\cdots$ co-former interactions, observed in the region between -30 and $-40 \mathrm{kJmol}^{-1}$, corresponding to the $\mathrm{O}-\mathrm{H} \cdots \mathrm{O}$ hydrogen bonds observed in the crystal structure, their strength enhanced through the cooperative effect of the linked interaction network.

The intermediate region of the plot containing moderate strength interactions is comprised of several weaker hydrogen bonds. Therse are generally considered favourable, however less influential than the main structure-directing, stronger interactions. When these are significant in number, their overall

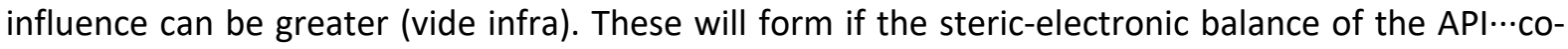
former assembly allows for the appropriate orientation and positioning of molecules but are less likely to influence the primary API...co-former assembly.

The final region at the top of the plot $\left(<10 \mathrm{kJmol}^{-1}\right)$ contains pairwise interactions comprised of many weak interactions. These are weaker and/or fewer in number than the moderate strength region resulting in lower overall interaction energies. These are likely to have lesser influence on structural arrangements, easily forming between adjacent molecules whose orientations are determined by the stronger interactions in the 'moderate' and 'strong' regions of the plot. 

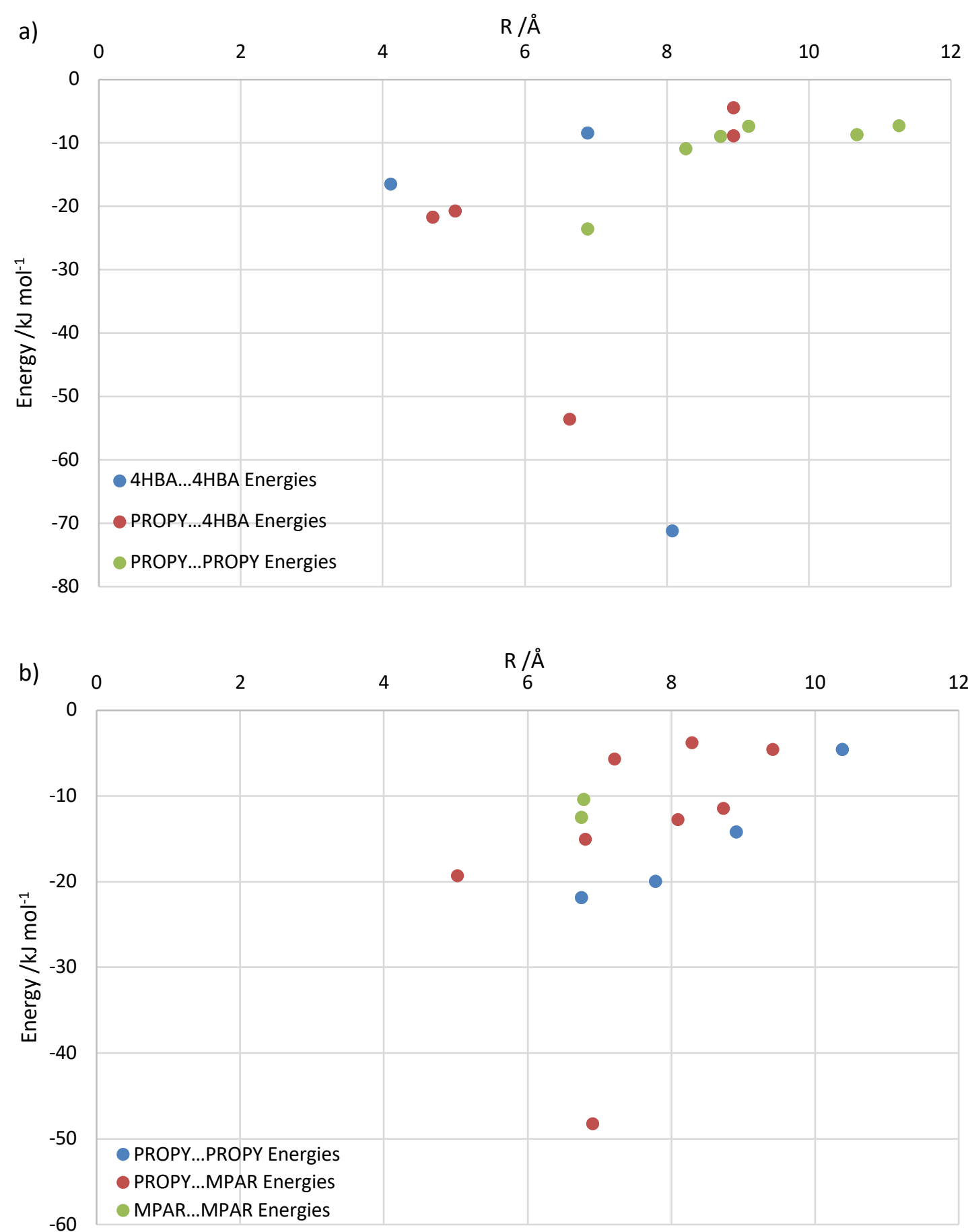


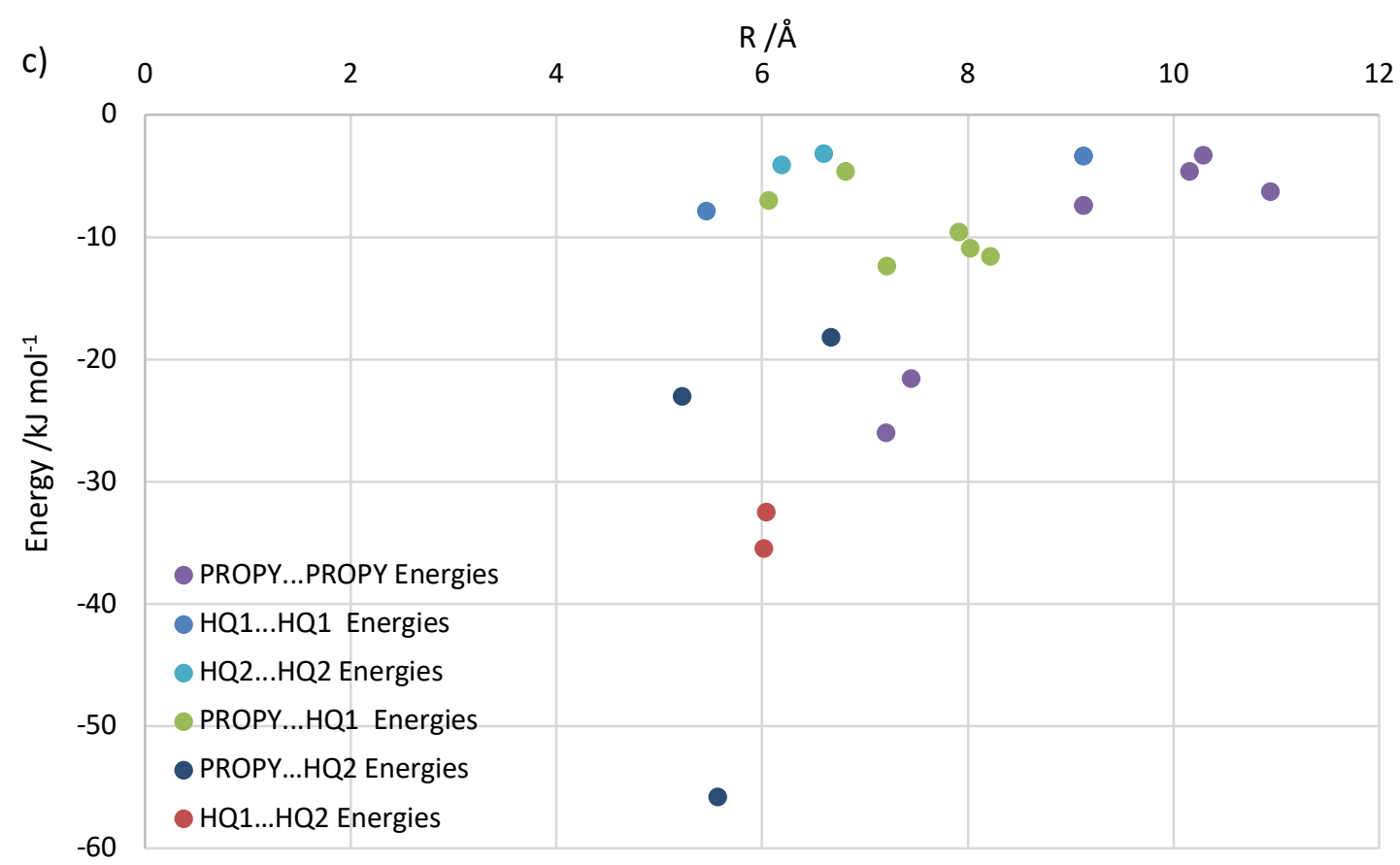

d)

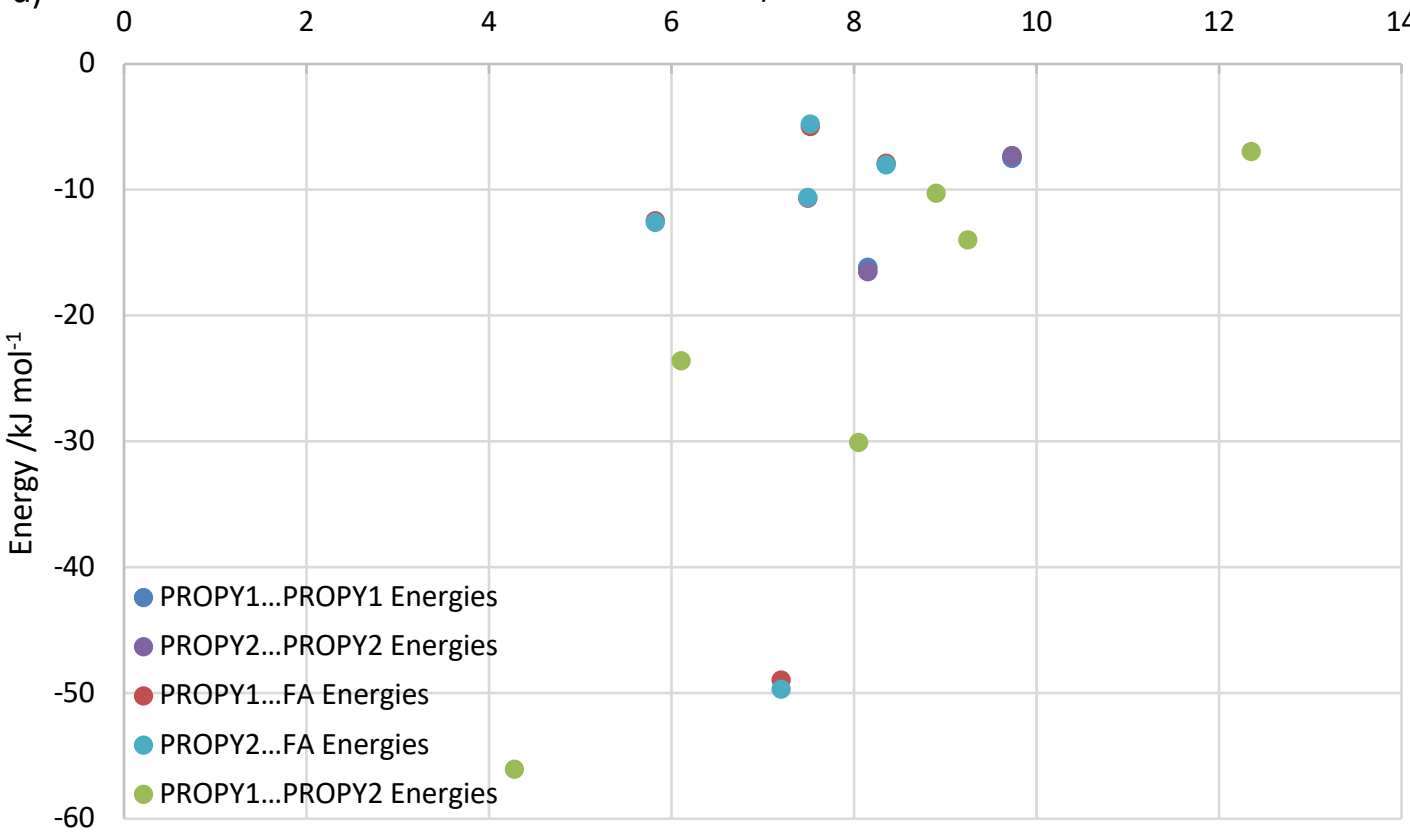

Figure 2. Interaction Energy Scatterplots determined from PIXEL calculations plotted against molecule $\cdots$ molecule centroid distances (R) for the PROPY co-crystals. a) PROPY_4HBA, b) PROPYMPAR, c) PROPY-HQ (note 1:2 ratio) and d) PROPY-FA (note 2:1 ratio). Weak interactions < -10 kJ $\mathrm{mol}^{-1}<$ Moderate interactions $<-30 \mathrm{~kJ} \mathrm{~mol}^{-1}<$ Strong interactions. 


\subsection{Aromatic Charge Distribution}

Several methods may be employed to integrate electron density and allocate charge to atomic centres (Stockholder partitioning,[45] Mulliken,[46] and Bader charges[18]). Full details of atomic charges, including Mulliken and Bader charges, are given in the Supporting Information Section 1.4, Tables S3 and S4. Stockholder partitioning is considered the most reliable,[45] and are therefore used herein. Figure 3 shows the Stockholder charges and $\rho\left(\mathbf{r}_{\mathrm{RCP}}\right)$ for the PROPY component of each system. A combined analysis of all these data makes it possible to compare between whole molecules and analyse areas of relative charge accumulation or depletion. The lowest $\rho\left(\mathbf{r}_{\mathrm{RCP}}\right)$ of the 5-membered PROPY ring is for PROPY-HQ $\left(0.320 e \AA^{-3}\right)$, where a greater charge accumulation is likely to result from increased hydrogen bonding. In contrast to the single strong hydrogen bond in the other systems, two hydrogen bonds form to the carbonyl and hence the density here might be expected to be larger.

In contrast to the extended network seen throughout the PROPY-HQ system, PROPY-MPAR exhibits no further strong hydrogen bonds from MPAR and henceforms discrete two-component units. This does not allow for distribution of the charge beyond the two molecules, and consequently the greatest $\rho\left(\mathbf{r}_{\mathrm{RCP}}\right)$ of the 5-membered ring is observed in PROPY-MPAR $\left(0.393 e \AA^{-3}\right)$. Similarly, the phenyl ring $\rho\left(\mathbf{r}_{\mathrm{RCP}}\right)$ is the largest seen across the four systems. Both PROPY-4HBA and PROPY-FA display a degree of extended charge distribution through a network of hydrogen bonds within discrete units: Propy4HBA in a 4-membered unit (Propy $\cdots 4$ HBA $\cdots 4$ HBA $\cdots$ PROPY) and PROPY-FA in a 3-membered unit

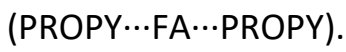




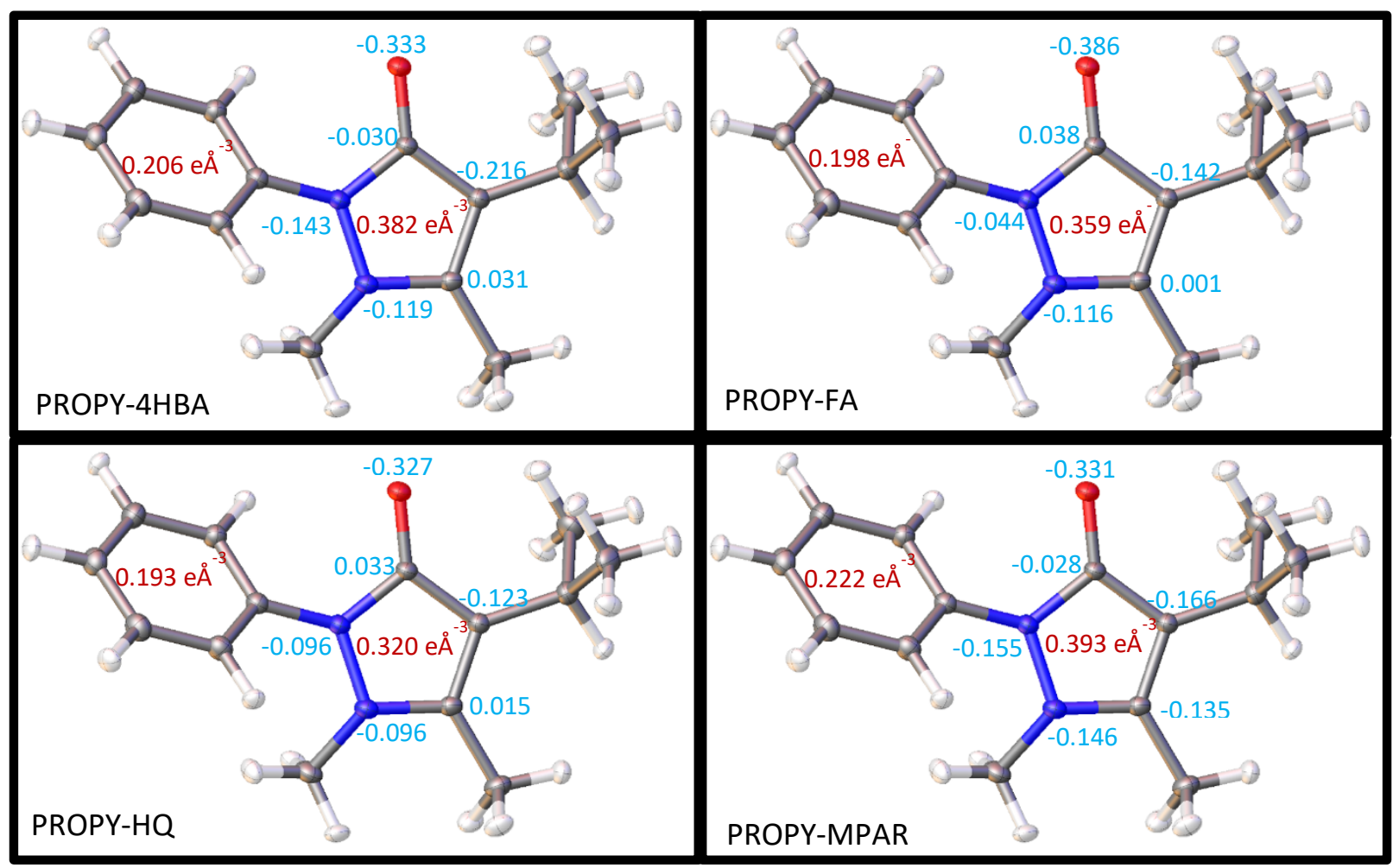

Figure 3. Atomic charges (blue) and $\rho(r)$ (red) at the RCPs for PROPY in the four systems, using Stockholder atomic partitioning for charges.

With this exception, a conventional comparison of charges and $\rho\left(\mathbf{r}_{\mathrm{RCP}}\right)$ does not reveal significant differences between the systems. While $\rho\left(\mathbf{r}_{\mathrm{RCP}}\right)$ might represent a larger moiety in the molecule, it does not provide the necessary detail for a "whole molecule" approach to be adopted for comparison. For this reason, it is necessary to combine understanding of classical interactions derived from the experimental EDD with theoretical molecular pair analysis to gain appropriate insight into all aspects of interaction between molecules.

\subsection{Theoretical Interaction Energies and their Components}

Figure 3 (above) illustrates the potential of calculating and comparing molecule $\cdots$ molecule interactions rather than looking at individual molecules, or atoms, in isolation. Analysis of an energy (or other property) landscape at the boundary between different pairs of molecules in a co-crystal system readily highlights important interactions. However, the major advantage of this approach is the ability to deconstruct these total molecule $\cdots$ molecule interactions into the individual contribution from each of their energy components. These contributions to an overall energy ( $E_{\text {coul, }} E_{\text {disp }}$ and $E_{\text {rep }}$ ) in each system are presented in Figure 4. Full numerical data grouped according to strength can be seen 
in Supporting Information Section 2.1, Table S11. As $E_{\text {pol }}$ is non-additive the sum of polarisation terms is not a valid indicator or value for gross comparisons as conducted herein and so this term is excluded from comparisons reported in the rest of this study.

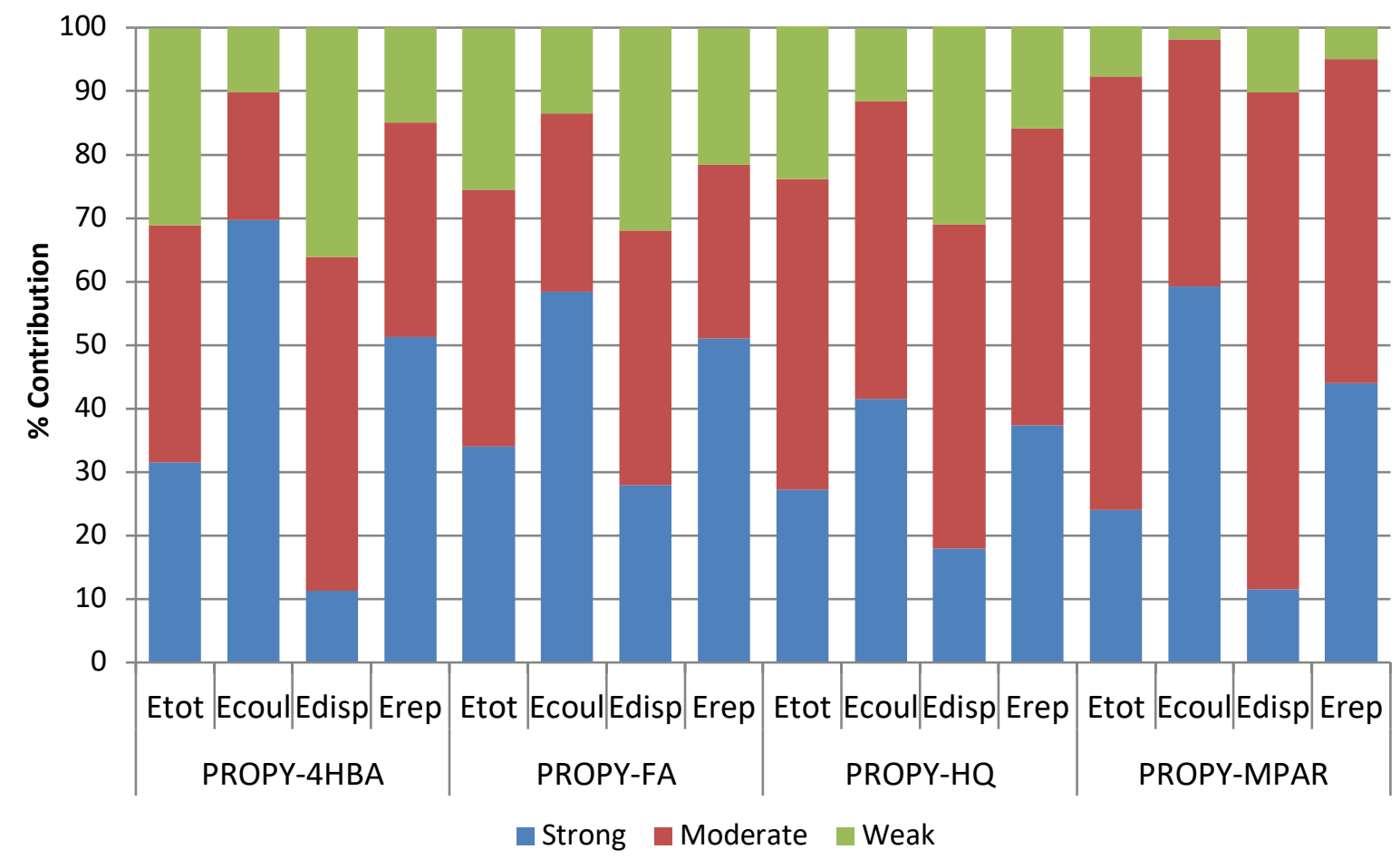

Figure 4. Contributions of the strong, moderate and weak (blue, red and green respectively) interactions to the Coulombic $\left(E_{\text {coul }}\right)$, dispersion $\left(E_{\text {disp }}\right)$, repulsion $\left(E_{\text {rep }}\right)$ and total energy $\left(E_{\text {tot }}\right)$ components of the four PROPY systems from PIXEL calculations.

For the strong interactions, dispersion energy $\left(E_{\text {dip }}\right)$ is a relatively small proportion of the total for all systems; PROPY-FA displays the greatest total dispersion energy contribution (28-35\%), with the remainder containing between $9-18 \%$ of the total energy in the system.

In all systems, the sum of the moderate strength interactions typically accounts for the greatest proportion of the total energy andexceeds the contribution of the strongest interactions. PROPY-HQ and PROPY-MPAR have a significantly greater percentage of the total energy contained within the moderate, compared to strong, interactions. PROPY-4HBA and PROPY-FA have a more equal distribution across the two groups, with moderate strength interactions being slightly more significant.

Weak interactions account for approximately $20-30 \%$ of the total energy in most systems. The exception to this is PROPY-MPAR, which contains less than $10 \%$ of the total interaction energy within these molecular pairings. The Coulombic component of these weaker interactions of PROPY-MPAR is 
also considerably smaller than observed in the other three systems (less than $2 \%$ of the total Coulombic energy in comparison to $13.5 \%$ in PROPY-FA).

\subsection{The Influence of Molecular Pair Type}

Table 1 categorises the molecular pair energies according to the participating molecules, separated into constituent energy components. As discussed MPAR contains no hydrogen bonding functionality beyond that involved in the API...MPAR primary interaction. The smaller co-former contribution and lower Coulombic energy value in comparison to the other systems is therefore expected.

The strongest interactions are highly favourable, contributing beneficially to the total energy and arise

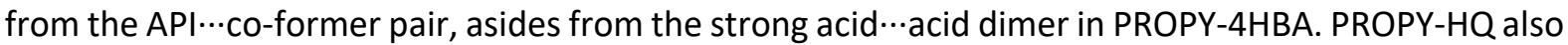
displays a large $E_{\text {coul }}$ arising from co-former...co-former interactions (as discussed previously, arising through the extended hydrogen bonding network). Neither PROPY-MPAR or PROPY-FA contain significant contributions from these interactions due to the lack of functionality available for interactions beyond the primary hydrogen bond.

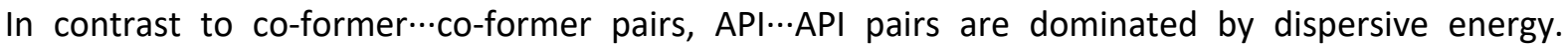

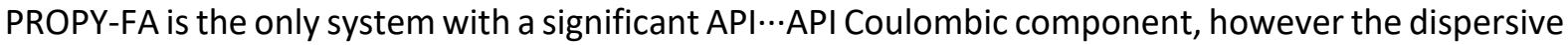
energy still dominates. PROPY-4HBA, -HQ and -MPAR all have similar Coulombic, dispersive and repulsive energies between PROPY molecules. These systems contain PROPY in a 1:1 stoichiometry and hence an equal number of PROPY molecules are present to form interactions. Accordingly, when two equivalents of PROPY are present (PROPY-FA) increased PROPY...PROPY contacts are observed.

Table 1. Interaction energies for the PROPY co-crystal systems obtained from PIXEL calculations,

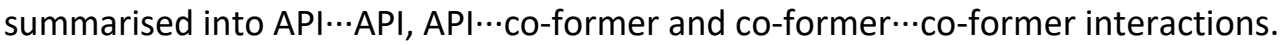

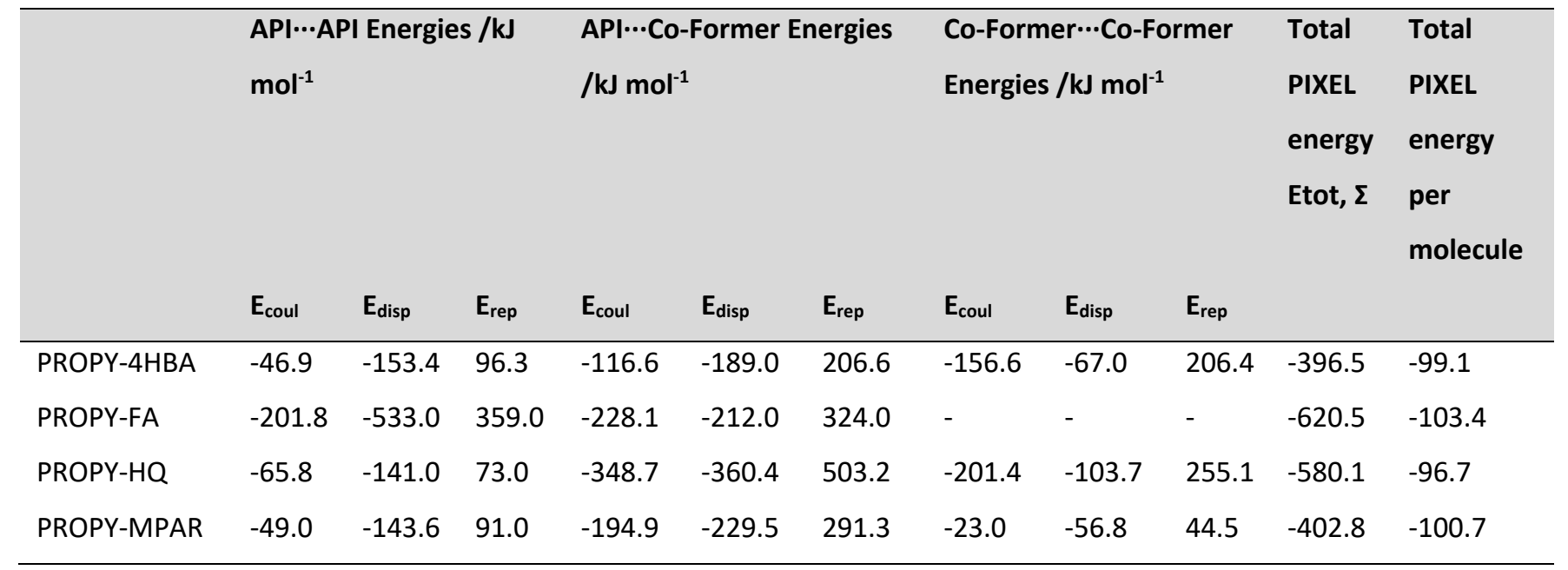




\subsection{Contributions of the Coulombic and Dispersion Energy Components}

Hydrogen bonds are the greatest contributor to experimental energies and primarily arise from the Coulombic energy contribution to the calculated molecular pair interactions, whilst other less obvious interactions presumably arise primarily from dispersive contributions. This hypothesis is discussed in the following specific examples (full data is in the Supporting Information Section 4):

In the case of PROPY-4HBA, the experimental analysis revealed that the acid $\cdots$ acid dimer involving 4HBA molecules is stronger than the PROPY-4HBA pairing, however the latter was identified as the stronger interaction from theoretical calculations. Inspection of the individual molecular pair energy

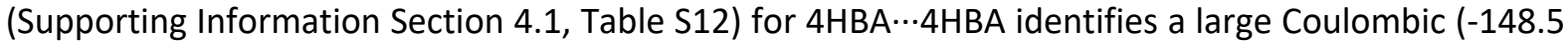
$\left.\mathrm{kJ} \mathrm{mol}^{-1}\right)$ and a small dispersive energy $\left(-23.1 \mathrm{~kJ} \mathrm{~mol}^{-1}\right)$ component. In comparison, the PROPY $\cdots 4 \mathrm{HBA}$ pair has a similar dispersive component $\left(-23 \mathrm{~kJ} \mathrm{~mol}^{-1}\right)$ but a significantly smaller $\mathrm{E}_{\text {coul }}\left(-74.8 \mathrm{~kJ} \mathrm{~mol}^{-1}\right)$. This greater Coulombic energy is identified from the experimental analysis and the total energy is greater than that of the energy calculated using the theoretical approaches.

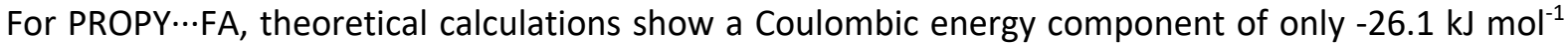
for the PROPY...PROPY interaction, with the total energy being dominated by dispersive forces (-68.2 $\left.\mathrm{kJ} \mathrm{mol}^{-1}\right)$. The PROPY ‥FA interaction, however, is dominated by the Coulombic energy $\left(-88.2 \mathrm{~kJ} \mathrm{~mol}^{-1}\right)$ with a small dispersive component $\left(-21.6 \mathrm{~kJ} \mathrm{~mol}^{-1}\right)$. Experimental energies are derived from hydrogen bonds which are principally Coulombic in nature. The differences in the components of the PROPY $\cdots F A$ and PROPY...PROPY interaction energies account for the differences in the experimental energies; the large dispersive forces in the PROPY...PROPY interaction are not considered and the experimental interaction energy appears considerably reduced.

As presented, the strongest interactions are generally dominated by the Coulombic component and the weakest by dispersion. However, the relative discrete values for dispersion energy are greatest in the moderate strength interactions. Dispersion energy is most likely to arise from $\pi \cdots \pi$ interactions, such as the aromatic co-former and PROPY rings. The electronic environment of the aromatic rings in PROPY are similar in all systems and therefore it is the co-former that will affect the dispersive forces more considerably. Lacking an aromatic ring, FA is structurally different to the other co-formers and this is reflected in the dispersion energy. Here the largest dispersion energy is still manifested in the moderate strength interactions, however the strong interactions also have a large dispersion energy component. This is significantly greater than observed in the three other systems. FA contains a series 
of double bonds which can form $\pi$-interactions to the aromatic rings of PROPY (no FA …FA interactions occur). The $\rho(\mathrm{r})$ in these double bonds is greater than that in the co-former aromatic rings (see Supporting Information Section 1.6., Table S10). Accordingly, PROPY-FA would be expected to have stronger dispersive forces arising from an increased electron density.

\section{Discussion}

\subsection{The Interplay Between Structure and Co-crystal Composition}

\subsubsection{Primary $\mathrm{O}-\mathrm{H} \cdots \mathrm{O}$ hydrogen bond}

As described in our previous study,[25] each co-crystal exhibits a primary $\mathrm{O}-\mathrm{H} \cdots \mathrm{O}$ hydrogen bond between the co-former hydroxyl or carboxylic acid functional group and PROPY and this can be assessed using two approaches. Study of the molecular graph in conjunction with the molecular pair

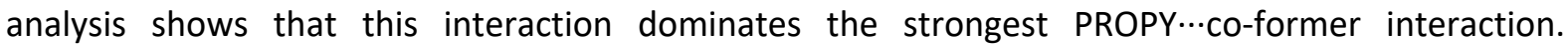
Experimental hydrogen bond estimations indicate similar energies for the hydroxyl...carbonyl hydrogen bonds in all systems displaying this motif $\left(\sim 50 \mathrm{~kJ} \mathrm{~mol}^{-1}\right)$, whilst the carboxylic acid $\cdots$ carbonyl (PROPY-FA) has a larger energy at almost $90 \mathrm{~kJ} \mathrm{~mol}^{-1}$. The breakdown of the energies in the fingerprint plots of PROPY and its co-crystals (Figure S17) are shown in Figure 5. 


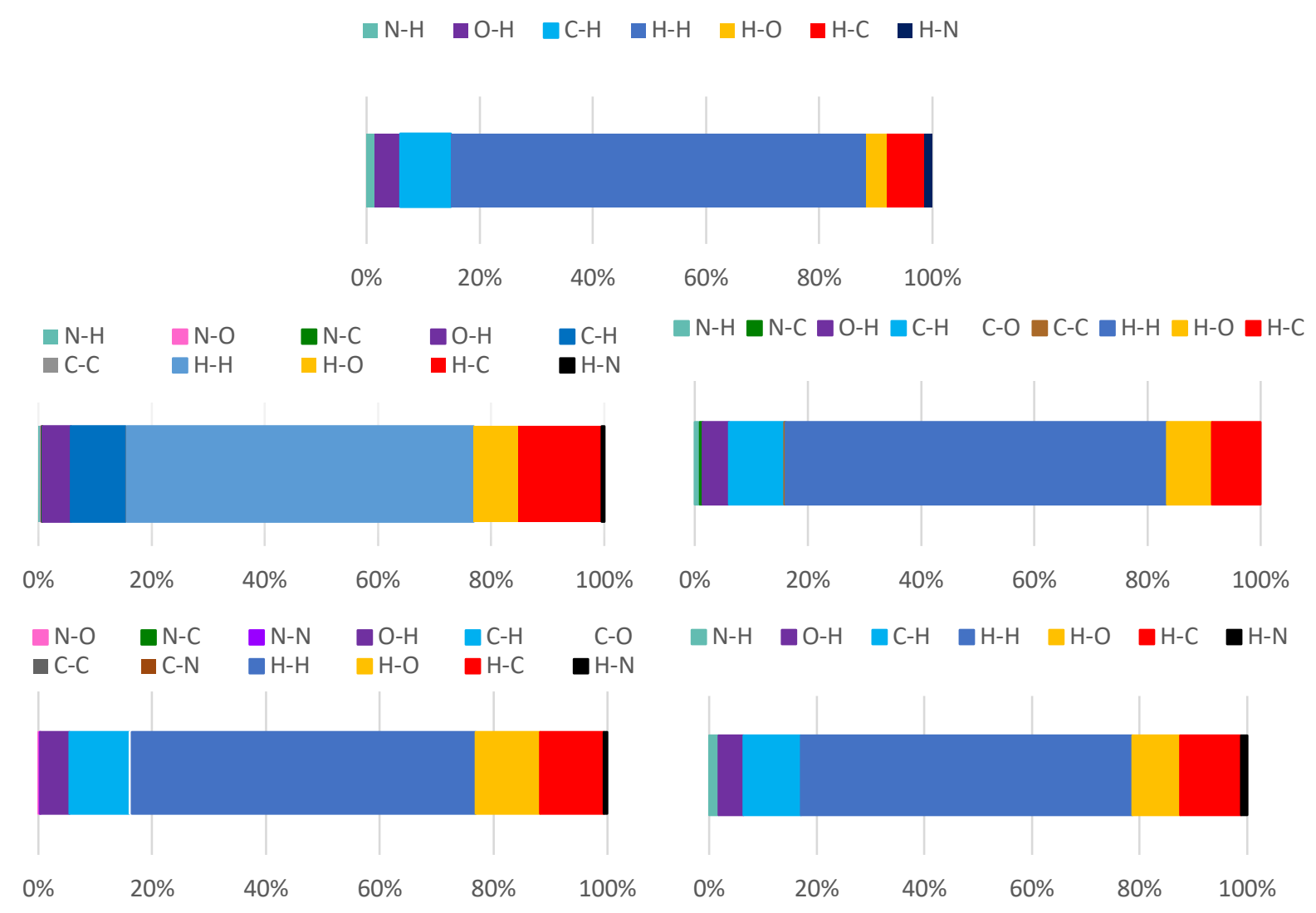

Figure 5. Interaction contributions for (a) PROPY and the four co-crystals; b) PROPY-4HBA, c) PROPY-FA, d) PROPY-HQ and e) PROPY-MPAR.

The addition of a co-former increases the relative proportion of non-C-H contacts due to a greater range of contacts possible from the additional functionalities present, i.e. hydrogen bonds and other interactions. Carboxylic acid *.carbonyl interactions (PROPY-FA) have a greater Coulombic energy than those from hydroxyl-containing co-formers. The dispersion energy components are similar in both cases due to the fact that Coulombic, not dispersion, energy determines hydrogen bond strength.

Figure 6 depicts molecular graphs obtained from the experimental model for the four systems, with bond critical points (BCPs) and ring critical points (RCPs) displayed and hydrogen bonds circled and shaded according to strength. It is clear from this visual depiction that there are differences in the interaction directions and strengths. Accordingly, $\rho(\mathrm{r})$ and $\nabla^{2} \rho(\mathbf{r})$ at the BCPs can be associated with molecular pairs (see Supporting Information Section 1.5., Tables S5-S9). The strong O-H $\cdots O$ O hydrogen bond occurs between the molecules in the molecular pair with the strongest interaction energy and therefore dominates the molecular pair interaction in each co-crystal.

However, unlike standard geometric analysis where a short interaction is assumed to be strong, and with almost linear angles, we can see that in the case of PROPY-FA and PROPY-HQ this is not always 
the case. The shorter O-H $\cdots$ O hydrogen bond $\left(r_{i j}=1.55 \AA, E=-87.75 \mathrm{~kJ} \mathrm{~mol}^{-1}\right)$ in PROPY-FA has a smaller interaction energy that its equivalent in PROPY-HQ $\left(r_{i j} 1.60 \AA ̊, E=-101.09 \mathrm{~kJ} \mathrm{~mol}^{-1}\right)$.
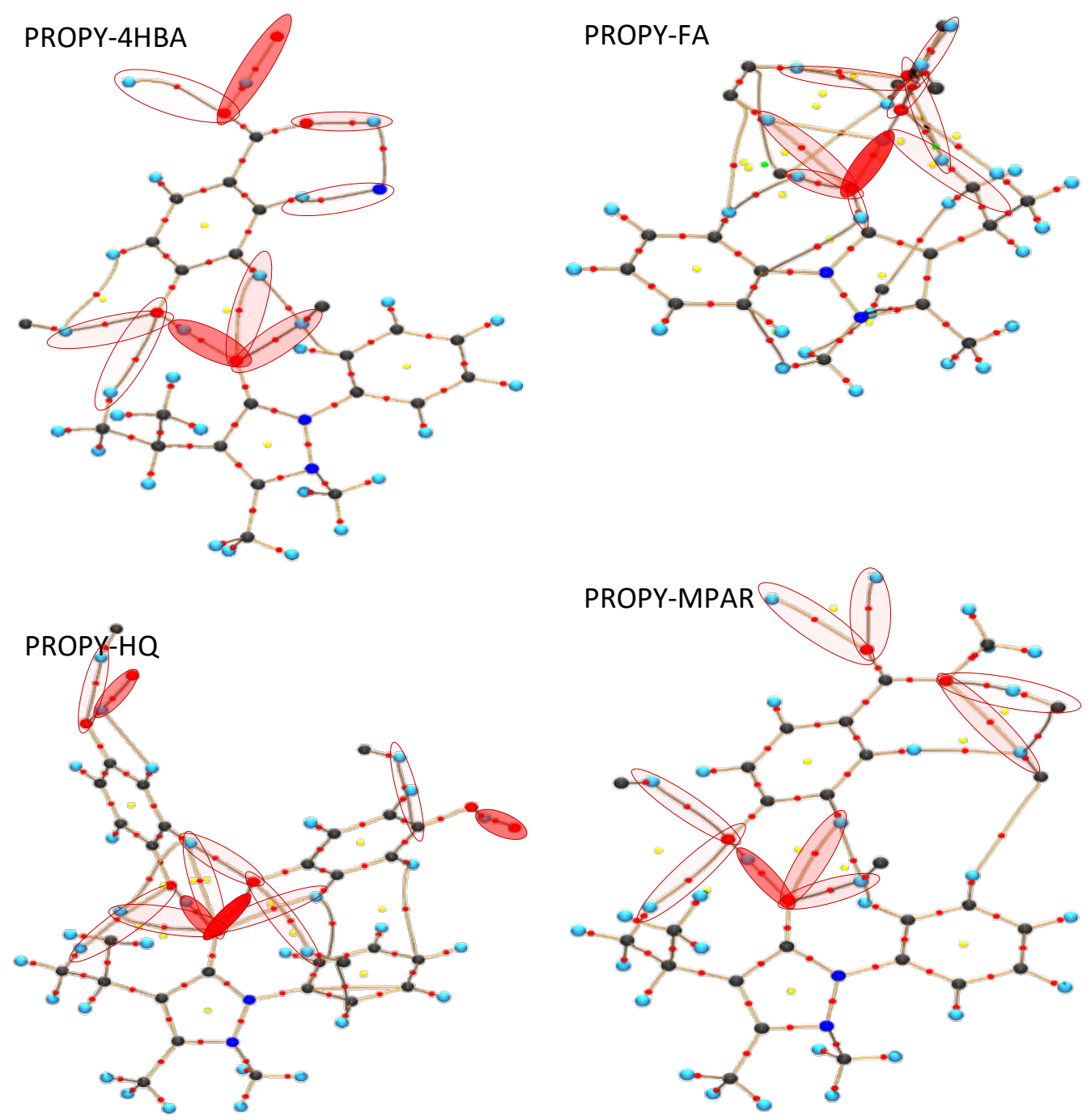

Figure 6. Molecular graph diagrams showing atomic nuclei (coloured according to atom type), connected by bond paths (gold) with BCP (red) and RCP (yellow) displayed. Interactions have been circled and shaded according to strength; a darker shading indicates a stronger interaction.

This can be rationalised due to the additional hydrogen bonds present within the molecular pairings in each co-crystal. Usually these are much weaker interactions and their contribution to the overall pairwise energy is minimal, however the presence of further interactions in PROPY-HQ create a cooperative effect, increasing the strength of the single $\mathrm{O}-\mathrm{H} \cdots \mathrm{O}$ hydrogen bond through the accumulation of electron density from a greater number of atoms. This provides some evidence for understanding the effects of structural diversity and stoichiometry and indicates differences between aromatic and non-aromatic systems. 


\subsubsection{Comparing Structural Similarities: Interactions and Assemblies}

PROPY-4HBA and PROPY-MPAR have the greatest degree of structural similarity and can therefore probe connections between similar co-formers and their intermolecular interactions. They exhibit similar RCP properties and atomic charges, illustrating how small structural changes in co-formers can correlate with the electronic distribution of PROPY (the hydrogen bond acceptor), rather than that of the co-formers themselves (exhibited by the Stockholder partitioned atomic charges and $\rho\left(\mathbf{r}_{\mathrm{RCP}}\right)$ in Figure 7). However, the ester group in place of a carboxylate in MPAR reduces hydrogen bonding functionality and this subtle difference terminates the interaction network, forming discrete twocomponent units. This could affect the redistribution of charge beyond the two molecules - a modest accumulation of charge is observed in the PROPY, where the $\rho\left(r_{\mathrm{RCP}}\right)$ of the aromatic rings in PROPYMPAR have the highest values across all systems (see Supporting Information Section 1.6., Table S10). PROPY-4HBA displays the second highest $\rho\left(r_{\mathrm{RCP}}\right)$ for both the PROPY rings. Though discrete units of PROPY-4HBA are generated, as in PROPY-MPAR, they are comprised of four molecules (2 PROPY-4HBA adducts connected via the carboxylic acid dimer). Although charge can be redistributed throughout the hydrogen bonding network, it can also be received via the dimerised 4HBA molecule, thus countering any charge redistribution. The increased number of constituent atoms enables more redistribution of the charge, however MPAR and 4HBA have identical RCP densities while the carboxylate dimer interaction has relatively large $\rho\left(\mathbf{r}_{\mathrm{RCP}}\right)$ values and is the dominant reason for the greater charge accumulation in PROPY. 


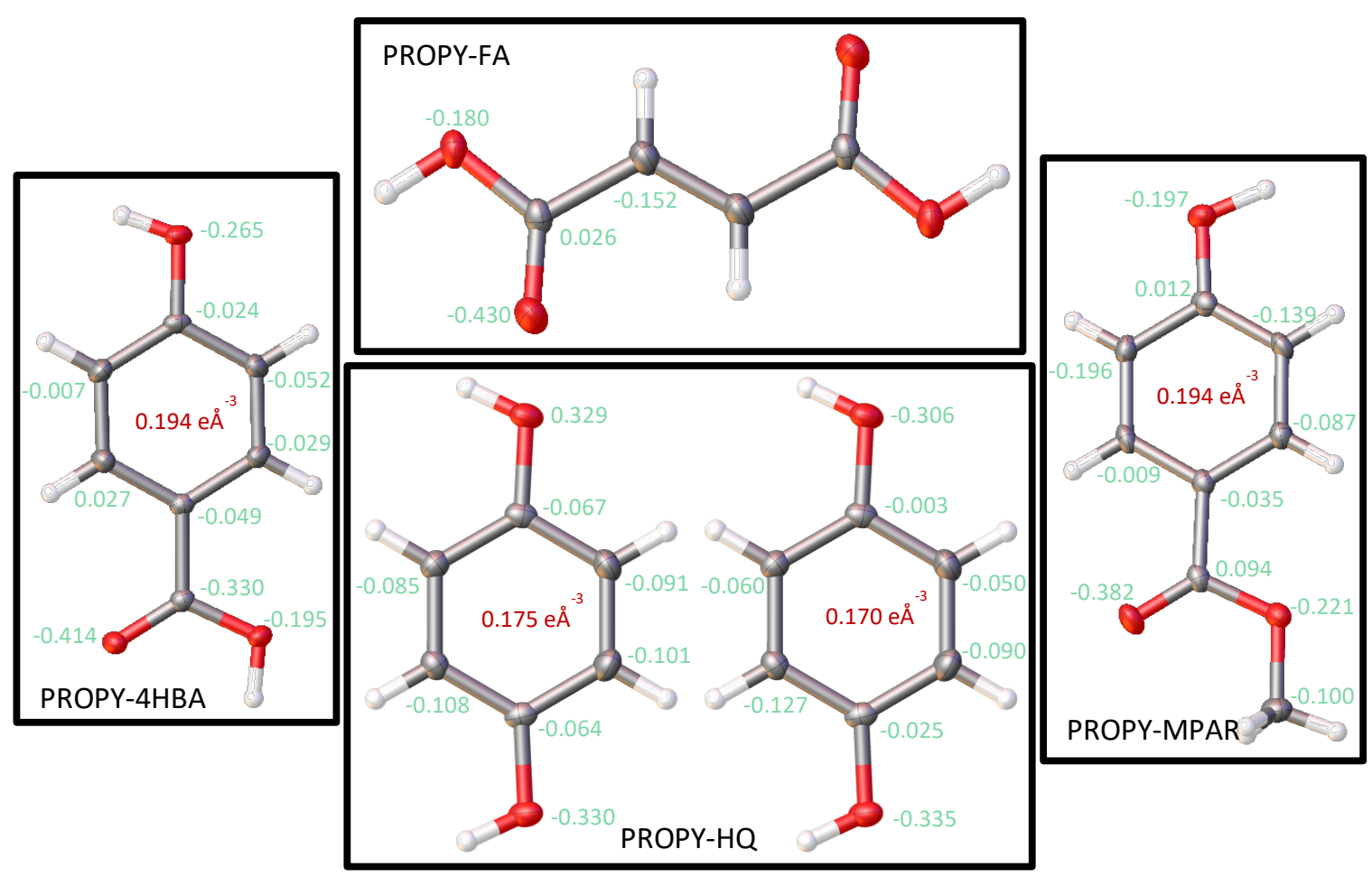

Figure 7. Atomic charges (blue) and $\rho(r)$ (red) at the RCPs for the co-former molecules in the four systems, using Stockholder atomic partitioning for charges.

Theoretical molecular pair interactions show the energy components of PROPY...PROPY pairs in both systems to be similar, however PROPY...co-former and co-former...co-former pairs highlight the subtle differences in the structures. PROPY...4HBA pairs have a smaller Coulombic energy component than the MPAR equivalent (-116.6 vs $\left.-194.9 \mathrm{~kJ} \mathrm{~mol}^{-1}\right)$, however the 4HBA $\cdots 4 \mathrm{HBA}$ pairs are considerably stronger than MPAR $\cdots$ MPAR pairings $\left(-156.6 \mathrm{vs}-23.0 \mathrm{~kJ} \mathrm{~mol}^{-1}\right)$. The strongest theoretical molecular pair interaction has a slightly increased energy in PROPY-4HBA in comparison to that of PROPY-MPAR, although the experimental hydrogen bond energy is contrary.

These comparisons and contrasting features of the two systems consider the similarities of the two co-former molecules related to the para-substituted aromatic ring, however the difference in the functionality opposite the primary hydrogen bond is apparent. Both PROPY-MPAR and PROPY-4HBA display a strong PROPY...co-former pairwise interaction of a similar strength. This is also similar to that in PROPY-HQ, however differs in the structurally diverse PROPY-FA.

\subsubsection{Contrasting Structural Diversity}

PROPY-FA is a structurally diverse member and in addition to the contrasting primary hydrogen bond $\left(\mathrm{O}-\mathrm{H}_{\text {carboxylic acid }} \cdots \mathrm{O}\right.$ vs. $\mathrm{O}-\mathrm{H}_{\text {hydroxyl }} \cdots \mathrm{O}_{\text {carbonyl }}$ ) can be used to probe the differences between aromatic and linear co-formers. PROPY-FA displays a moderate $\rho\left(r_{R C P}\right)$ for both PROPY rings despite forming discrete 
2:1 units with no further hydrogen bond network, such as that seen in PROPY-4HBA. Each FA interacts with two PROPY molecules, thereby reducing the amount of charge available to each PROPY as FA is essentially halved in its capacity to contribute. Additionally, the carboxylic acid $\cdots$ carbonyl hydrogen bond is strong with a high $\rho\left(r_{\mathrm{RCP}}\right)$ value, indicating the relative accumulation of a substantial amount of charge.

PROPY-FA shows the greatest disparity between experimental and theoretical values of interaction energies, which can be explained when looking at the structure. FA is the only co-former which does not contain an aromatic ring and possesses no additional hydrogen bond functionalities to form favourable hydrogen bonds (besides that partaking in the primary $\mathrm{O}-\mathrm{H}_{\text {carboxylic acid }} \cdots \mathrm{O}_{\text {carbonyl }}$ hydrogen bond). As a result, many of the forces present are less atom directed (e.g. $\pi-\pi$ stacking) and cannot be detected in this analysis, leading to differences in the quantification of the interactions derived from the two approaches. The other three systems (PROPY-4HBA, PROPY-HQ and PROPY-MPAR) show good correlations between their energy components, contributions and physicochemical properties however in many respects, PROPY-FA is observed as an anomaly.

\subsubsection{Co-crystal Stoichiometry}

$H Q$, like 4HBA and MPAR, is a para-substituted aromatic co-former molecule however it exists in a 1:2 co-crystal with PROPY. The second hydroxyl group of $\mathrm{HQ}$ creates a sterically smaller functional group in comparison to the carboxylic acid/ester groups of $4 \mathrm{HBA}$ and MPAR, providing an opportunity to investigate rationalisation of the stoichiometric ratio preference based on a steric vs. electronic argument.

An increased ratio of $\mathrm{HQ}$ permits a greater number of interactions, generating a larger total energy for the co-crystal system $\left(-580.1 \mathrm{~kJ} \mathrm{~mol}^{-1}\right)$. Electronic factors seek to maximise the interactions and distribution of charge, which the additional hydroxyl functionality of HQ facilitates. HQ does not dimerise with itself (as with $4 \mathrm{HBA}$ ), instead it forms moderate co-former $\cdots$ co-former $\mathrm{O}-\mathrm{H}$ hydroxyl ${ }^{\cdots} \mathrm{O}_{\text {hydroxyl }}$ hydrogen bonds $\left(-35.5 \mathrm{~kJ} \mathrm{~mol}^{-1}\right)$ which extend the hydrogen bond network and stabilise the system through distribution of charge. The presence of two hydroxyl groups interacting with the PROPY carbonyl is possible due to their relatively small size enabling both to position sufficiently close and in a favourable orientation to form an interaction. This is made more favourable as each hydroxyl group is both donating and receiving electron density through the hydrogen bonding network, so there is no charge accumulation and resultant repulsive force. 


\subsection{Insights into Physical Properties}

Analysis of co-crystal intermolecular interactions provides extensive insight into the interplay between different forces present in the crystalline lattice. This provides a basis for relating structural information to trends and characteristics of solid-state physicochemical properties. For heat-induced or degradation breakdown of a molecular solid to occur the network of intermolecular interactions holding the molecules together in the lattice must be broken, enabling the transition from an ordered solid state to a less ordered liquid phase or ordered parent phases. Understanding this phenomenon for a multicomponent complex is more involved, however it has the potential to provide key understanding that will enable rationalisation and tuning of physiochemical properties through the co-crystalisation approach.

\subsubsection{Melting Point}

Analysis of the interplay of these intermolecular interactions i.e. molecule $\cdots$ molecule energy components and also into individual hydrogen bonds, can provide a rationale behind the trend in melting points. Table 2 shows the rank order of the melting point as determined by DSC.

Table 2. The first row provides the rank order of melting point from highest to lowest colour coded by co-crystal. The rank order, using the same colour code, is then given for comparison of key groupings in the interaction energy analysis.

\begin{tabular}{|c|c|c|c|c|}
\hline Trend & Greatest & & & Least \\
\hline Melting Point $\left({ }^{\circ} \mathrm{C}\right)$ & $\begin{array}{l}\text { PROPY-HQ } \\
(133.9)\end{array}$ & $\begin{array}{l}\text { PROPY-4HBA } \\
(133.4)\end{array}$ & $\begin{array}{l}\text { PROPY-FA } \\
(108.0)\end{array}$ & $\begin{array}{l}\text { PROPY-MPAR } \\
(70.2)\end{array}$ \\
\hline Strongest pairwise interactions & PROPY-FA & PROPY-HQ & PROPY-4HBA & PROPY-MPAR \\
\hline API...co-former hydrogen bonds & PROPY-HQ & PROPY-FA & PROPY-4HBA & PROPY-MPAR \\
\hline Coulombic energy per molecule & PROPY-HQ & PROPY-4HBA & PROPY-MPAR & PROPY-FA \\
\hline Dispersion energy per molecule & PROPY-FA & PROPY-MPAR & PROPY-4HBA & PROPY-HQ \\
\hline
\end{tabular}

For comparison and rationalisation, the rank order of different groupings of interaction energies is also presented (for absolute values, see Supporting Information Section 5, Table S15). Logically, the strongest interactions require the most energy to break and these define the barrier to melting. As 
the ranking of melting points does not agree with that of total experimentally derived energies (Figure 8), a more complex situation clearly exists. PROPY-HQ exhibits the highest melting point and the largest total number of interactions, in conjunction with a large Coulombic energy from strong and moderate interactions. The Coulombic energy factor is particularly important, being significantly larger than the other systems, and is indicative of a stronger and therefore more stable complex as previously discussed.

While the strongest interactions dictate the initial energy barrier, the moderate strength interactions also play an important role. These are much weaker than the strongest interactions, but greater in number - PROPY-MPAR has the "weakest" total moderate energy interactions (see Supplementary Information, Table S11) and exhibits the lowest melting point of the series, despite having a greater total energy than PROPY-FA (when considering that there are two FA molecules per PROPY). The total energy is dominated by the Coulombic component which is higher in PROPY-MPAR and so the number of moderate interactions in relation to the energy can be considered a significant factor in melting point ranking.

Experimental total energies

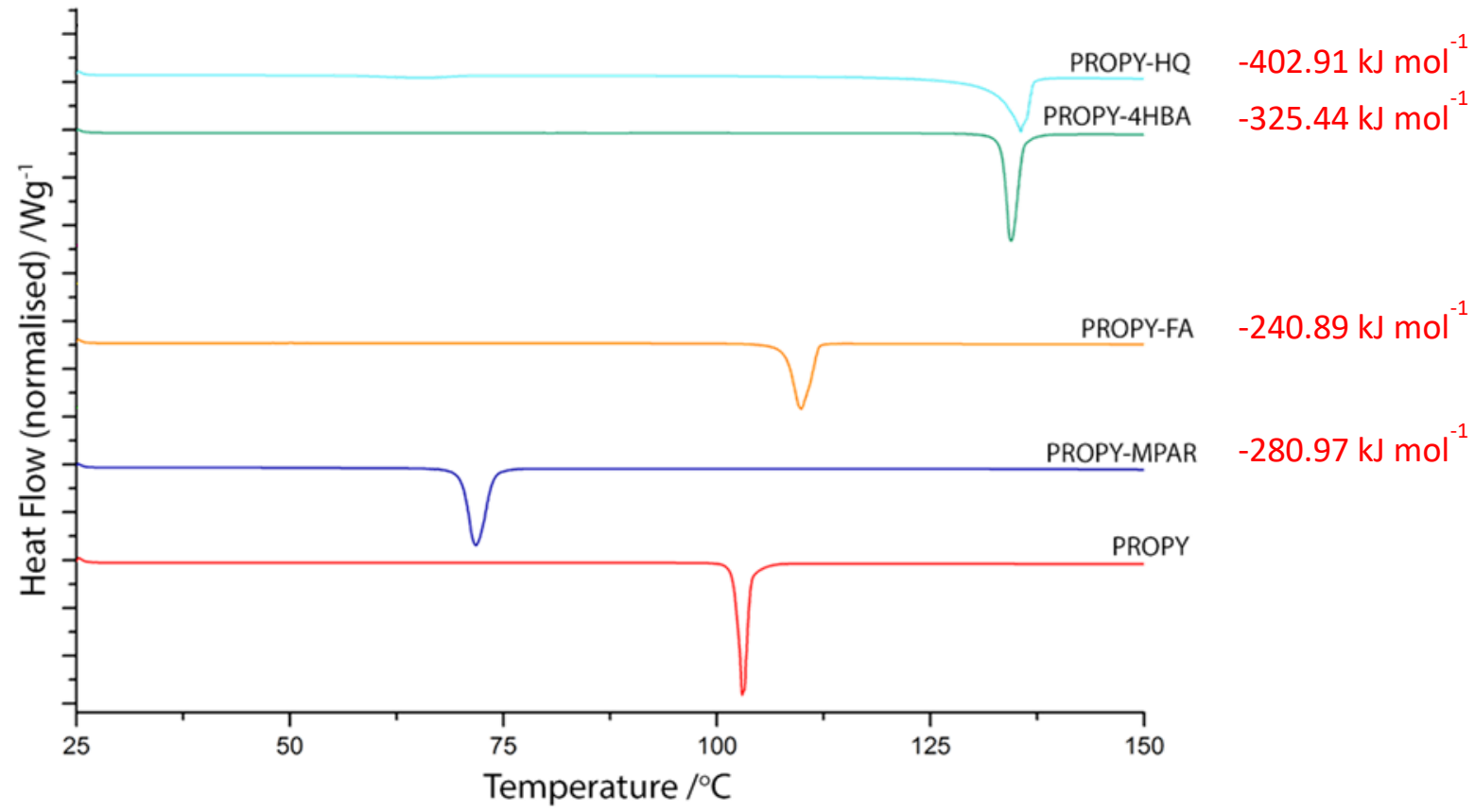

Figure 8. DSC plots, measured at a heating rate of $10^{\circ} \mathrm{C} \mathrm{min}^{-1}$ from 25 to $150{ }^{\circ} \mathrm{C}$, indicating melting points for all co-crystals and the PROPY parent. The corresponding total experimental energy is displayed (red) for each co-crystal system.

Strong co-former $\cdots$ co-former hydrogen bonds are also observed in PROPY-4HBA and PROPY-HQ. The ranking of individual API...co-former hydrogen bonds from the EDD is in reasonable agreement with 
the melting point order, however PROPY-FA and PROPY-4HBA rank differently. As noted previously, the quantity and magnitude of individual API...co-former hydrogen bonds should be considered in balance. PROPY-HQ has four strong primary hydrogen bonds, PROPY-4HBA has three, while PROPY-FA and PROPY-MPAR only have one. While PROPY-FA requires a greater energy to break the single hydrogen bond, PROPY-4HBA has three similarly strong hydrogen bonds to break.

A further aspect to consider is the relative composition of the total energy for each system. Theoretical analysis of PROPY-4HBA highlights that the strongest interactions have a much larger Coulombic energy, requiring a greater energy to break. The overall Coulombic energy per molecule can be seen to increase across the series and this is reflected in the melting point trend. Coulombic and dispersion energy components appear to have a negative correlation with one another (vide supra) and consideration of the trend in dispersion energy indicates that the system with the highest melting point exhibits the lowest dispersion energy.

Larger, more negative, charges on the PROPY carbonyl group oxygen could be expected to relate to a higher melting point. However, the greatest of these was seen in PROPY-FA (in all three atomic partitioning methods) which does not exhibit the highest melting point. The structural diversity seen in FA compared to 4HBA, HQ and MPAR (vide supra) causes reduced aromaticity and a far less degree of delocalisation of the $\pi$-electrons than in aromatic rings. The observed trends and relationships can therefore be considered to be reasonably appropriate for systems containing aromatic co-formers with structural similarity to those presented herein. However, further investigation would be required to confirm whether this is applicable to all aromatic co-formers, or how the relationships vary.

\subsubsection{Solubility}

Solid state stability has been linked to lower aqueous solubility,[47] resulting from the stronger association of molecules in the solid state and so it follows that intermolecular interaction energy and solubilty would be inversely related. However, a similar intermolecular analysis based solely on strength and composition does not fully explain or corroborate trends in the measured solubility (or dissolution), as it does not provide a holistic or comprehensive understanding. Only a simplistic understanding of the attenuating influence of intermolecular interactions on solution properties can be garnered from this analysis. Some of the strongest interactions are observed in PROPY-HQ despite its high observed solubility, whereas those with weaker interactions e.g. PROPY-MPAR, display poorer solubilities and varying dissolution rates (see Table 3).

This unexpected trend in solubility could be due to several reasons. Firstly, to assess solubility based on an analysis of intermolecular interaction energies of co-crystals would likely mean an assumption 
that it is dependent on all interactions breaking simultaneously. This might not be the case as, for example, a 2 step process could occur whereby the weaker interactions break first but retain the API...co-former conjugate, which then has to be broken. Secondly, strong co-former...co-former

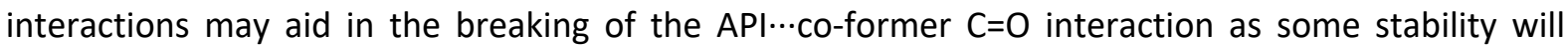
remain. Therefore PROPY-HQ and PROPY-4HBA (see Table 1) have an advantage and 'release' the PROPY more easily than PROPY-FA and PROPY-MPAR, which have a greater contribution to the overall interaction energy from the API...co-former $\mathrm{C}=\mathrm{O}$ interactions and are thus more reliant on this interaction to retain stability.

Table 3. Solubility of PROPY and co-crystals pH 7.5 phosphate buffer at room temperature.

\begin{tabular}{lll}
\hline Sample & Solubility $/ \mathrm{mg} \mathrm{mL}^{-1}$ & Ratio to API \\
\hline PROPY & 2.71 & 1 \\
PROPY-4HBA & 2.19 & 0.81 \\
PROPY-FA & 1.82 & 0.67 \\
PROPY-HQ & 3.14 & 1.16 \\
PROPY-MPAR & 1.48 & 0.55 \\
\hline
\end{tabular}

\subsubsection{Dissolution Rate}

Melting only involves factors concerning the arrangement of molecules in the lattice and therefore the observed interactions are the determining factors for this property. However dissolution involves the breaking apart of molecular constructs due to a disturbance to the solid state lattice caused by a solvent. A proposed mechanism is one whereby the solvent forms interactions to the solute, disrupting the intermolecular interactions of the solute. Therefore, the respective strength of solute intermolecular interactions will influence the rate at which they are broken. The dissolution rate plot (Figure 9) demonstrates the strong positive correlation between solubility and dissolution, with only one visible outlier in PROPY-4HBA. A justification for this is in the strong 4HBA acid-acid dimer, which creates a kinetic barrier to lattice breakdown, resulting in a slower initial dissolution rate than expected. Conversely in the thermodynamically dependent solubility process, time allows for the breaking of this dimer. 


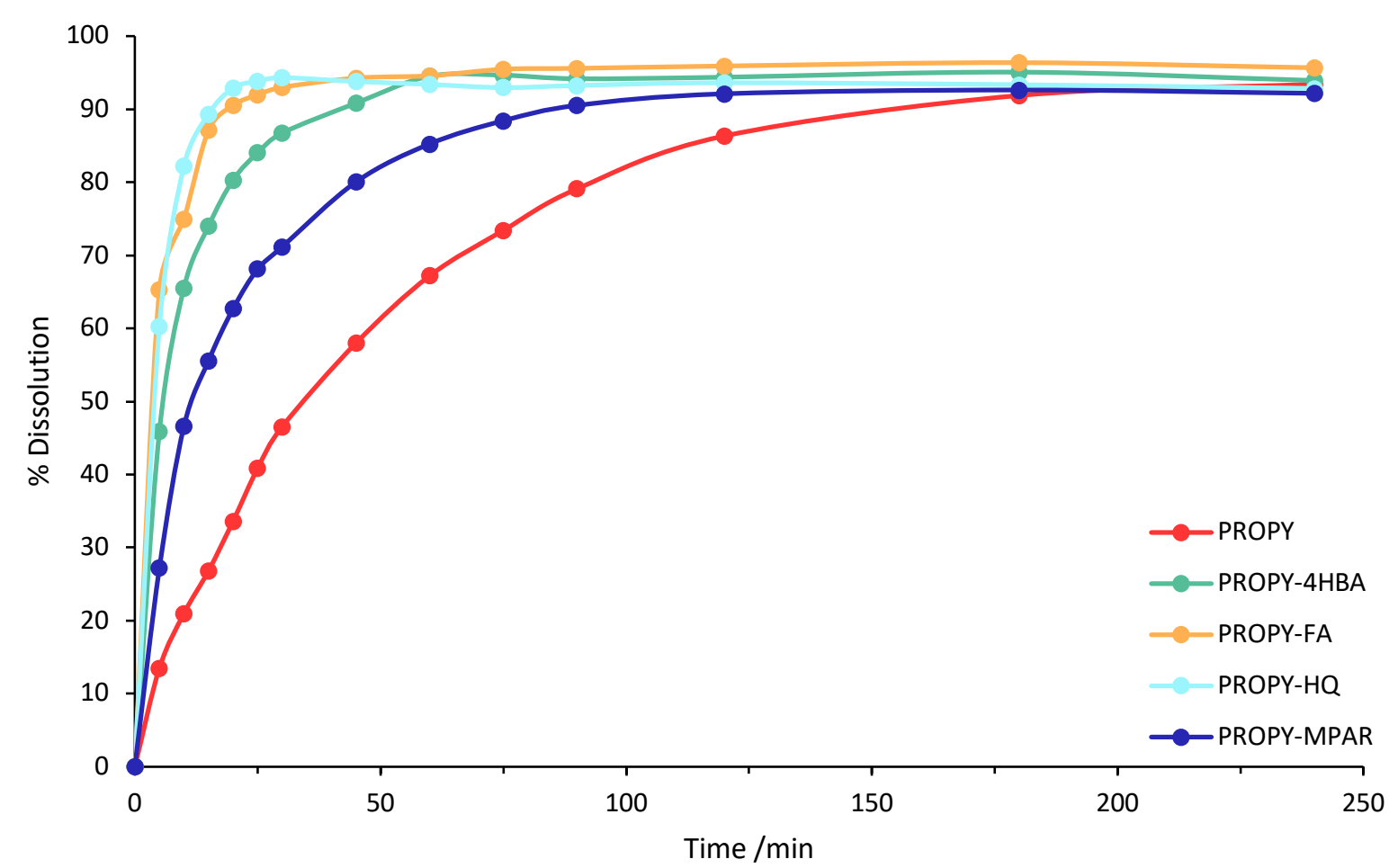

Figure 9. Dissolution rate profile of PROPY and co-crystals carried out over 240 min experiment time at $37^{\circ} \mathrm{C}$ in $\mathrm{pH} 7.5$ phosphate buffer.

\subsubsection{Stability}

All four materials analysed showed no indication of dissociation or breakdown in stability tests (see Supporting Information Section 7, Figures S16 and S17). Interaction analysis has shown that all four co-crystals contain strong, highly favourable interactions, both at the atom -..atom level via hydrogen bonding and at the molecular level between molecular pairs. Numerous other, generally weaker, interactions occur between molecules in the solid-state and they are not necessarily all beneficial. $\mathrm{A}$ multitude of "trade-offs" must occur when placing molecules adjacent to each other and there will be attractive forces that are beneficial, as well as repulsive forces, which incur penalties. All of these must be taken into account when considering total energetic contributions. In this study no significant destabilising interactions have been evidenced, which supports the observed stability.

\section{Conclusions}

At electronic resolution the charge distribution in molecules can be evaluated. Along with quantumbased approaches to the deconvolution of the molecular pair interaction energies into their components, this helps to understand the nature of the interactions, the significance to the overall pairwise energy and can some correlations made with measured physicochemical properties. Applying this method to co-crystals helps to shed light on the influence of the co-former, and the differences 
that arise from subtle changes in the co-former structure, such as stoichiometry and physicochemical properties. This approach identifies that there are other important factors, beyond the primary hydrogen bonds and motifs, that are fundamental in controlling these solid state assemblies and their behaviour. This methodology can provide further rationalisation of physiochemical properties, particularly when a systematic approach is taken.

Melting point is seen to be dominated by the Coulombic energy term, with a negative correlation to the dispersion energy component, seen primarily in strong and moderate strength interactions. Coformers tune the primary hydrogen bond and influence the structural formation, which in turn influences physicochemical properties. The juxtaposition of these competing favourable interactions with the 'penalties' incurred in other parts of the lattice is only understandable when traditional intermolecular interactions vs. molecular pairings are decomposed into their contributing components (Coulombic and dispersion energies). Partial rationalisation of solution properties is possible from a purely energetic point of view, with the knowledge that there are other influencing factors, such as solvent $\cdots$ solute interactions. Solubility can be influenced by the disruption of weak solute $\cdots$ solute interactions and retained stability of co-former $\cdots$ co-former pairs, over time, that promote the release and observed increase of PROPY in solution. Dissolution rate correlates positively with solubility, with the exception of PROPY-4HBA, which suggests the breaking of strong 4HBA-4HBA dimers provides an initial barrier to its kinetically dependent dissolution.

Whilst many of the strong interactions identified can be considered "structure directing" and "property defining", they are not necessarily "structure defining" and do not completely govern the resulting crystal structure arrangement. Further analysis of the interplay between experimental electron density distribution and the energetic values of intermolecular interactions this dictates, and theoretical molecular pair interaction energies has the potential to shed light on the factors influencing co-crystal structure composition.

\section{Conflicts of interest}

There are no conflicts to declare.

\section{Acknowledgements}

This research was funded by the Science and Engineering Research Council of A*STAR (Agency for Science, Technology and Research), Singapore. L. K. Mapp and J. Cadden thank A*STAR for a research fellowship jointly funded by the A*STAR Research Attachment Programme (ARAP) and The University of Southampton. 


\section{References}

[1] G.R. Desiraju, Supramolecular synthons in crystal engineering - a new organic synthesis, Angew. Chem., Int. Ed. Engl. 34 (1995) 2311-2327. https://doi.org/10.1002/anie.199523111.

[2] S. Aitipamula, R. Banerjee, A.K. Bansal, K. Biradha, M.L. Cheney, A.R. Choudhury, G.R. Desiraju, A.G. Dikundwar, R. Dubey, N. Duggirala, P.P. Ghogale, S. Ghosh, P.K. Goswami, N.R. Goud, R.R.K.R. Jetti, P. Karpinski, P. Kaushik, D. Kumar, V. Kumar, B. Moulton, A. Mukherjee, G. Mukherjee, A.S. Myerson, V. Puri, A. Ramanan, T. Rajamannar, C.M. Reddy, N. Rodriguez-Hornedo, R.D. Rogers, T.N.G. Row, P. Sanphui, N. Shan, G. Shete, A. Singh, C.C. Sun, J.A. Swift, R. Thaimattam, T.S. Thakur, R. Kumar Thaper, S.P. Thomas, S. Tothadi, V.R. Vangala, N. Variankaval, P. Vishweshwar, D.R. Weyna, M.J. Zaworotko, Polymorphs, salts, and cocrystals: What's in a name?, Cryst. Growth Des. 12 (2012) 2147-2152. https://doi.org/10.1021/cg3002948.

[3] N. Schultheiss, A. Newman, Pharmaceutical cocrystals and their physicochemical properties, Cryst. Growth Des. 9 (2009) 2950-2967. https://doi.org/10.1021/cg900129f.

[4] N.K. Duggirala, M.L. Perry, Ö. Almarsson, M.J. Zaworotko, Pharmaceutical cocrystals: Along the path to improved medicines, Chem. Commun. 52 (2016) 640-655. https://doi.org/10.1039/c5cc08216a.

[5] N.J. Babu, A. Nangia, Solubility advantage of amorphous drugs and pharmaceutical cocrystals, Cryst. Growth Des. 11 (2011) 2662-2679. https://doi.org/10.1021/cg200492w.

[6] Z. Rahman, A.S. Zidan, R. Samy, V.A. Sayeed, M.A. Khan, Improvement of physicochemical properties of an antiepileptic drug by salt engineering, AAPS PharmSciTech. 13 (2012) 793-801. https://doi.org/10.1208/s12249-012-9800-9.

[7] Y. Gao, H. Zu, J. Zhang, Enhanced dissolution and stability of adefovir dipivoxil by cocrystal formation., J. Pharm. Pharmacol. 63 (2011) 483-90. https://doi.org/10.1111/j.2042-7158.2010.01246.x.

[8] S. Aitipamula, A.B.H. Wong, P.S. Chow, R.B.H. Tan, Cocrystallization with flufenamic acid: Comparison of physicochemical properties of two pharmaceutical cocrystals, CrystEngComm. 16 (2014) 5793-5801. https://doi.org/10.1039/c3ce42182a.

[9] V.R. Vangala, P.S. Chow, R.B.H. Tan, Characterization, physicochemical and photostability of a co-crystal involving an antibiotic drug, nitrofurantoin, and 4hydroxybenzoic acid, CrystEngComm. 13 (2011) 759-762. https://doi.org/10.1039/c0ce00772b.

[10] M.K. Stanton, A. Bak, Physicochemical properties of pharmaceutical co-crystals: A case study of ten AMG 517 co-crystals, Cryst. Growth Des. 8 (2008) 3856-3862. https://doi.org/10.1021/cg800173d.

[11] N. Shan, M.L. Perry, D.R. Weyna, M.J. Zaworotko, Impact of pharmaceutical cocrystals: the effects on drug pharmacokinetics., Expert Opin. Drug Metab. Toxicol. 10 (2014) 1255-71. https://doi.org/10.1517/17425255.2014.942281.

[12] C.M. Reddy, G. Rama Krishna, S. Ghosh, Mechanical properties of molecular crystals Applications to crystal engineering, CrystEngComm. 12 (2010) 2296-2314. https://doi.org/10.1039/c003466e.

[13] N. Blagden, S.J. Coles, D.J. Berry, Pharmaceutical co-crystals-are we there yet?, CrystEngComm. 16 (2014) 5753-5761. https://doi.org/10.1039/c4ce00127c. 
[14] C.B. Aakeröy, T.K. Wijethunga, J. Desper, Crystal engineering of energetic materials: co-crystals of Ethylenedinitramine (EDNA) with modified performance and improved chemical stability., Chemistry. 21 (2015) 11029-37.

https://doi.org/10.1002/chem.201501721.

[15] P. Vishweshwar, J.A. Mcmahon, M.J. Zaworotko, Crystal Engineering of Pharmaceutical Co-crystals, in: Front. Cryst. Eng., wiley, 2006: pp. 25-49. https://doi.org/10.1002/0470022612.ch2.

[16] P.T.A. Galek, L. Fábián, W.D.S. Motherwell, F.H. Allen, N. Feeder, Knowledge-based model of hydrogen-bonding propensity in organic crystals, Acta Crystallogr. Sect. B Struct. Sci. 63 (2007) 768-782. https://doi.org/10.1107/S0108768107030996.

[17] S.A. Moggach, W.G. Marshall, D.M. Rogers, S. Parsons, How focussing on hydrogen bonding interactions in amino acids can miss the bigger picture: A high-pressure neutron powder diffraction study of $\varepsilon$-glycine, CrystEngComm. 17 (2015) 5315-5328. https://doi.org/10.1039/c5ce00327j.

[18] R. F. W. Bader, Atoms in Molecules: A Quantum Theory, Oxford University Press, Clarendon Press, 1990. https://global.oup.com/academic/product/atoms-inmolecules-9780198558651?cc=in\&lang=en\& (accessed October 29, 2019).

[19] E. Madicines Agency, Committee for Medicinal Products for Human Use (CHMP) Committee for Medicinal Products for Veterinary Use (CVMP) Reflection paper on the use of cocrystals of active substances in medicinal products, 2015. www.ema.europa.eu/contact (accessed October 21, 2019).

[20] Regulatory Classification of Pharmaceutical Co-Crystals | FDA, (n.d.). https://www.fda.gov/regulatory-information/search-fda-guidancedocuments/regulatory-classification-pharmaceutical-co-crystals (accessed October 21, 2019).

[21] W.T. A Harrison, H.S. Yathirajan, S. Bindya, H.G. Anilkumar, Escitalopram oxalate: coexistence of oxalate dianions and oxalic acid molecules in the same crystal, (n.d.). https://doi.org/10.1107/S010827010605520X.

[22] A. Karagianni, M. Malamatari, K. Kachrimanis, Pharmaceutical cocrystals: New solid phase modification approaches for the formulation of APIs, Pharmaceutics. 10 (2018). https://doi.org/10.3390/pharmaceutics10010018.

[23] G. Bolla, A. Nangia, Pharmaceutical cocrystals: Walking the talk, Chem. Commun. 52 (2016) 8342-8360. https://doi.org/10.1039/c6cc02943d.

[24] Drug Trials Snapshot: Entresto | FDA, (n.d.). https://www.fda.gov/drugs/drugapprovals-and-databases/drug-trials-snapshot-entresto (accessed October 21, 2019).

[25] L.K. Mapp, S.J. Coles, S. Aitipamula, Design of cocrystals for molecules with limited hydrogen bonding functionalities: Propyphenazone as a model system, Cryst. Growth Des. 17 (2017) 163-174. https://doi.org/10.1021/acs.cgd.6b01399.

[26] C.R. Groom, I.J. Bruno, M.P. Lightfoot, S.C. Ward, The Cambridge Structural Database, Acta Crystallogr. Sect. B. 72 (2016) 171-179. https://doi.org/10.1107/s2052520616003954.

[27] S. Grabowsky, A. Genoni, H.B. Bürgi, Quantum crystallography, Chem. Sci. 8 (2017) 4159-4176. https://doi.org/10.1039/c6sc05504d.

[28] A. Nangia, G.R. Desiraju, Supramolecular Synthons and Pattern Recognition, in: 1998: pp. 57-95. https://doi.org/10.1007/3-540-69178-2_2.

[29] 2013 CrystalClear-SM Expert 3.1 b27, Rigaku, No Title, (n.d.).

[30] P. Coppens, X-ray Charge Densities and Chemical Bonding, International Union of 
Crystallography, Oxford University Press, 1997.

https://doi.org/10.1107/s0021889898015325.

[31] E. Espinosa, E. Molins, C. Lecomte, Hydrogen bond strengths revealed by topological analyses of experimentally observed electron densities, Chem. Phys. Lett. 285 (1998) 170-173. https://doi.org/10.1016/S0009-2614(98)00036-0.

[32] M.A. Spackman, How Reliable Are Intermolecular Interaction Energies Estimated from Topological Analysis of Experimental Electron Densities?, Cryst. Growth Des. 15 (2015) 5624-5628. https://doi.org/10.1021/acs.cgd.5b01332.

[33] Crystal Explorer (Version 17.5), Wolff, S. K., Grimwood, D. J., McKinnon, J. J., Turner, M. J., Jayatilaka, D., Spackman, M. A. (2012) University of Western Australia, Perth., (n.d.).

[34] M.J. Frisch, G.W. Trucks, H.B. Schlegel, G.E. Scuseria, M.A. Robb, J.R. Cheeseman, G. Scalmani, V. Barone, G.A. Petersson, H. Nakatsuji, X. Li, M. Caricato, A. V Marenich, J. Bloino, B.G. Janesko, R. Gomperts, B. Mennucci, H.P. Hratchian, J. V Ortiz, A.F. Izmaylov, J.L. Sonnenberg, Williams, F. Ding, F. Lipparini, F. Egidi, J. Goings, B. Peng, A. Petrone, T. Henderson, D. Ranasinghe, V.G. Zakrzewski, J. Gao, N. Rega, G. Zheng, W. Liang, M. Hada, M. Ehara, K. Toyota, R. Fukuda, J. Hasegawa, M. Ishida, T. Nakajima, Y. Honda, O. Kitao, H. Nakai, T. Vreven, K. Throssell, J.A. Montgomery Jr., J.E. Peralta, F. Ogliaro, M.J. Bearpark, J.J. Heyd, E.N. Brothers, K.N. Kudin, V.N. Staroverov, T.A. Keith, R. Kobayashi, J. Normand, K. Raghavachari, A.P. Rendell, J.C. Burant, S.S. Iyengar, J. Tomasi, M. Cossi, J.M. Millam, M. Klene, C. Adamo, R. Cammi, J.W. Ochterski, R.L. Martin, K. Morokuma, O. Farkas, J.B. Foresman, D.J. Fox, Gaussian 09, (2016).

[35] C.F. Mackenzie, P.R. Spackman, D. Jayatilaka, M.A. Spackman, CrystalExplorer model energies and energy frame-works: extension to metal coordination compounds, organic salts, solvates and open-shell systems, 4 (2017) 575-587. https://doi.org/10.1107/S205225251700848X.

[36] J.J. McKinnon, A.S. Mitchell, M.A. Spackman, Hirshfeld surfaces: A new tool for visualising and exploring molecular crystals, Chem. - A Eur. J. 4 (1998) 2136-2141. https://doi.org/10.1002/(SICI)1521-3765(19981102)4:11<2136::AIDCHEM2136>3.0.CO;2-G.

[37] M.A. Spackman, J.J. McKinnon, Fingerprinting intermolecular interactions in molecular crystals, CrystEngComm. 4 (2002) 378-392. https://doi.org/10.1039/b203191b.

[38] J.J. McKinnon, M.A. Spackman, A.S. Mitchell, Novel tools for visualizing and exploring intermolecular interactions in molecular crystals, Acta Crystallogr. Sect. B Struct. Sci. 60 (2004) 627-668. https://doi.org/10.1107/S0108768104020300.

[39] J.J. McKinnon, D. Jayatilaka, M.A. Spackman, Towards quantitative analysis of intermolecular interactions with Hirshfeld surfaces, Chem. Commun. (2007) 38143816. https://doi.org/10.1039/b704980c.

[40] M.A. Spackman, D. Jayatilaka, Hirshfeld surface analysis, CrystEngComm. 11 (2009) 19-32. https://doi.org/10.1039/b818330a.

[41] A. Gavezzotti, Towards a realistic model for the quantitative evaluation of intermolecular potentials and for the rationalization of organic crystal structures. Part I. PhilosophyElectronic Supplementary Information (ESI) is available: the GAUSSIAN input files, the Pixe, CrystEngComm. 5 (2003) 429. https://doi.org/10.1039/b311831b. 
[42] A. Gavezzotti, Towards a realistic model for the quantitative evaluation of intermolecular potentials and for the rationalization of organic crystal structures. Part II. Crystal energy landscapes, CrystEngComm. 5 (2003) 439-446. https://doi.org/10.1039/b311836c.

[43] A. Gavezzotti, Calculation of intermolecular interaction energies by direct numerical integration over electron densities. 2. An improved polarization model and the evaluation of dispersion and repulsion energies, J. Phys. Chem. B. 107 (2003) 23442353. https://doi.org/10.1021/jp022288f.

[44] A. Gavezzotti, Calculation of lattice energies of organic crystals: The PIXEL integration method in comparison with more traditional methods, Zeitschrift Fur Krist. 220 (2005) 499-510. https://doi.org/10.1524/zkri.220.5.499.65063.

[45] R.K. Roy, Stockholders charge partitioning technique. A reliable electron population analysis scheme to predict intramolecular reactivity sequence, J. Phys. Chem. A. 107 (2003) 10428-10434. https://doi.org/10.1021/jp035848z.

[46] R.S. Mulliken, Electronic population analysis on LCAO-MO molecular wave functions. I, J. Chem. Phys. 23 (1955) 1833-1840. https://doi.org/10.1063/1.1740588.

[47] D. Elder, R. Holm, Aqueous solubility: Simple predictive methods (in silico, in vitro and bio-relevant approaches), Int. J. Pharm. 453 (2013) 3-11.

https://doi.org/10.1016/j.ijpharm.2012.10.041. 